\title{
Self-Efficacy as the Mediator of the Relationship between Paternal Approval and Suicidal Ideation among Malaysian Adolescents
}

\section{Wu Shin Ling and Siti Nor Yaacob ${ }^{\mathrm{b}}$}

${ }^{a}$ Department of Psychology, Faculty of Social Sciences and Liberal Arts, UCSI University, Kuala Lumpur, Malaysia; ${ }^{b}$ Department of Human Development and Family Studies, Faculty of Human Ecology, Universiti Putra Malaysia, Serdang, Malaysia

*Corresponding author:

Wu Shin Ling

Department of Psychology, Faculty of Social

Sciences and Liberal Arts

UCSI University

Kuala Lumpur, Malaysia

Email address: shinling_wu@ @otmail.com 


\title{
Self-Efficacy as the Mediator of the Relationship between Paternal Approval and Suicidal Ideation among Malaysian Adolescents
}

\begin{abstract}
Suicide phenomenon among young generation has become a leading cause of death especially among adolescents. In Asian countries, suicidal rate is at an alarming state in which it has contributed around $60 \%$ of the world suicide cases. In order to prevent suicidal behavior, suicidal ideation need to be investigated as it happened prior any suicidal behavior. Thus, the aim of this research is to determine the relationships between paternal approval, selfefficacy and suicidal ideation among adolescents with self-efficacy as a mediator. Methods. This study used a cross-sectional research design where 682 adolescents aged between $14-17$ years old were selected from different high school in Malaysia. Multistage cluster sampling was used to recruit the participants using self-administered questionnaires. The instruments used were Network of Relationships - Relationship Quality Version subscale, General Self-Efficacy Scale and Negative subscale of the Positive and Negative Suicide Ideation Inventory. For analysis, Pearson's Correlation and SPSS macro by Preacher and Hayes was used to test the objectives of this study. Results. Negative correlation was found between paternal approval and suicidal ideation as well as between self-efficacy and suicidal ideation. These findings implied that adolescents who gained more approval from their father and had a higher level of self-efficacy were less likely to have suicidal thoughts. On the other hand, positive correlation was found between paternal approval and self-efficacy which demonstrated that fathers who gave more approval to their children will develop children with higher sense of self-efficacy. For the mediation model, the relationship between paternal approval and adolescents' suicidal ideation was successfully mediated by self-efficacy. The findings implied that paternal approval is important in adolescents' life as it is able to increase adolescents' self-efficacy which then is able to reduce the tendency of having suicidal thoughts. Conclusions. The current study has identified two important factors namely paternal approval and self-efficacy that were significantly correlated with adolescents' suicidal ideation. Adolescents are less likely to develop suicide thoughts when their father imposes less restrictions and give more supports to what they want to do in life. Gaining high approval from fathers also helps in increasing adolescents' level of self-efficacy. Therefore, intervention dealing with adolescents' suicidal ideation should include parenting education for parents especially for fathers and also enhancing adolescents' self-efficacy.
\end{abstract}

Keywords: adolescents, parental approval, self-efficacy, suicidal ideation

\section{Introduction}

According to the World Health Organization (WHO), approximately 800,000 people died due to suicide every year. Based on WHO's statistic, suicidal behaviour is at its critical stage as there is one person committed suicide every 40 seconds (WHO, 2018). In Malaysia, past statistic revealed that the suicidal rate had increased $60 \%$ in the past few years (Roslan, 2016). These statistics indicated that suicidal phenomena is a serious issue that requires the 
attention of the society. However, due to the sensitivity of this issue, many cases are underreported where the cases are classified as accident or other causes of death (Sipalan, 2012). Thus, study should focus on the factors predicting suicidal ideation in order to prevent this phenomenon.

Past study found that prior committing suicide, a person tends to have suicidal thoughts (Ibrahim, Amit, Che Din, \& Ong, 2017). Suicide ideation is defined as the thoughts and thinking one has about ending his or her own life (Nock et al., 2008). Past study indicated that suicidal ideation is the strongest predictor for suicide attempt to occur (Wan \& Leung, 2010). Since suicidal ideation is an important factor in predicting suicidal behavior, this study aimed to investigate the factors affecting suicidal ideation.

Suicidal ideation is associated with the environment and self-factors. Environment factors include the relationship with parents while self-factors include self-efficacy. Social Cognitive Theory (Bandura, 1986) indicated that the environment and self-factors affect someone's behavior where individual learns from their social environments while processing what they learnt through their cognitive abilities. Individual with good relationship with parents are better in coping and regulating negative thoughts. Thus, a positive relationship with parents is associated with greater self-efficacy which is able to lower the risk of suicidal ideation.

Past study found that supportive parents are important for positive developmental outcomes among adolescents, including lowering the risk of suicide (Taliaferro \& Muehlenkamp, 2014). In Asian communities, strong emotional ties between parent and children are highly valued which reflects the core familial values within the SEA region, including Malaysia (Gill \& Gopal, 2010). Students who experienced poor parental relationship have an increased risk of both suicidal ideation and suicidal attempts (You, Chen, Yang, Zhou, \& Qin, 2014).

Self-efficacy is another important factor in predicting suicidal ideation. It is a cognitive element about the beliefs that one is able to cope with a large variety of stressors (Bandura, 1977). McNamara (2012) highlighted that adolescents who have higher level of self-efficacy are able to connect positively with their social environment which protected them from suicidal risk. This was further supported by Feng and Chen (2015) who indicated that individuals with high level of self-efficacy were able to overcome stress easily which safeguarded them from having suicidal thoughts.

In recent decade, father has taken a major role in adolescents' life where there are large involvement in children's life (Palkovitz, Trask, \& Adamsons, 2014). Due to the shift of father's role, this research aimed to determine the relationships between paternal approval, self-efficacy and suicidal ideation among adolescents. Specifically, self-efficacy as the mediator of the relationship between paternal approval and suicidal ideation was examined. Thus, this study hypothesized (a) positive correlation between paternal approval and selfefficacy, (b) negative correlation between self-efficacy and suicidal ideation, (c) negative correlation between paternal approval and suicidal ideation, and lastly (d) self-efficacy would mediate the relationship between paternal approval and suicidal ideation. 


\section{Methods}

\section{Research Design}

Cross-sectional research design was used in this study. Multistage cluster sampling was used to recruit 682 adolescents who are currently studying in high school. Questionnaires which are self-administered were distributed to the participants and collected on the same day. The participants' age was between 14 to 17 years old where the mean age is 15.34 . The participants comprised of 378 (55.4\%) female and 304 (44.6\%) male adolescents.

\section{Instruments}

All of the instruments used in this study has been pilot tested and yield good reliability. The Network of Relationships - Relationship Quality Version (Buhrmester \& Furman, 2009) subscale was used to measure paternal approval. It has 3 items rated on 5-point Likert-type scale, ranging from 1 (Never or hardly at all) to 5 (Always or extremely much). Sample item include "How often does this person praise you for the kind of person you are?". This instrument yield high reliability with Cronbach's alpha value at .79 . Higher score indicated high level of approval from father.

General Self-Efficacy Scale (Schwarzer \& Jerusalem, 1995) was used to measure adolescents' self-efficacy. It has 10 items rated on 4-point Likert-type scale, ranging from 1 (Not at all true) to 4 (Exactly true). The reliability of this instrument is .80. Sample item include "I can always manage to solve difficult problems if I try hard enough.". Higher score indicated higher level of self-efficacy.

Suicidal ideation was assessed using the Negative subscale of the Positive and Negative Suicide Ideation Inventory (Osman et al., 1998). It consists of 8-items rated on 5-point Likert-type scale, ranging from 0 (None of the time) to 4 (Most of the time). However, due to the sensitivity of this issue in Malaysia, 2 items from the Suicidal Ideation Inventory Negative Scale was requested to be omitted by the Ministry of Education of Malaysia. These 2 items are "Thought about killing yourself because you could not find a solution to a personal problem?" and "Felt so lonely or sad you wanted to kill yourself so that you could end your pain?". The reliability of this instrument is .82. Sample item include "Seriously considered killing yourself because you could not live up to the expectations of other people?". Higher score indicated higher level of suicidal ideation.

\section{Data Analysis}

For analysis, correlations between paternal approval, self-efficacy and suicidal ideation among adolescents were analyzed using Pearson's Correlation. Meanwhile, the mediating effect of self-efficacy on the relationship between paternal approval and suicidal ideation among Malaysian adolescents was tested using SPSS macro by Preacher and Hayes.

\section{Results}

Based on the Pearson's Correlation analysis, significant correlations were found between paternal approval, self-efficacy and suicidal ideation among adolescents. Paternal approval ( $\mathrm{r}$ 
$=-.094, \mathrm{p} \leq .05)$, and suicidal ideation as well as between self-efficacy $(\mathrm{r}=-.141, \mathrm{p} \leq .001)$ and suicidal ideation were found to be negatively correlated as displays in Table 1 . These findings implied that adolescents who gained more approval from their father and obtained a higher sense of self-efficacy were less likely to have suicidal thoughts. On the other hand, positive correlation $(\mathrm{r}=.277, \mathrm{p} \leq .001)$ was found between paternal approval and selfefficacy which demonstrated that fathers who gave more approval to their children will develop children with higher sense of self-efficacy.

Table 1: Correlations between the study variables $(\mathrm{N}=682)$

\begin{tabular}{lllll}
\hline & & \multicolumn{2}{r}{$\mathrm{r}$} \\
No. & Variable & 1 & 2 & 3 \\
\hline 1. & Paternal approval & - & - & - \\
2. & Self-efficacy & $.277 * * *$ & - & - \\
3. & Suicidal Ideation & $-.094 *$ & $-.141 * * *$ & - \\
\hline \multicolumn{5}{r}{ Note. ${ }^{* * *} \mathrm{p}<.001,{ }^{*} \mathrm{p}<.05$}
\end{tabular}

For the mediation model, self-efficacy successfully mediated the relationship between paternal approval and adolescents' suicidal ideation. The indirect effect was significant where the Sobel test indicated a mediation effect $(Z=-2.88, p \leq .01)$. Bootstrapping analysis showed that the path coefficients for indirect effect of paternal approval on suicidal ideation through self-efficacy were -.049 (95\% CI: -.091, -.019). The findings implied that paternal approval is important in adolescents' life as it is able to increase adolescents' self-efficacy. When adolescents' self-efficacy increases, it will help to reduce the tendency of having suicide thoughts.

\section{Discussion, Conclusion, and Implications}

The findings of the study supported the hypotheses in this study where (a) positive correlation were found between paternal approval and self-efficacy, (b) negative correlation were found between self-efficacy and suicidal ideation, (c) negative correlation were found between paternal approval and suicidal ideation, (d) self-efficacy mediated the relationship between paternal approval and suicidal ideation.

This study found that adolescents were less likely to develop suicidal thoughts when their father gives them more approval by imposes less restrictions and gives more supports to what they want to do in life. This is supported by Luca, Wyman, and Warren (2012) who found that supportive father tends to lower the risk of suicidal ideation among adolescents. In addition, Piña-Watson, Castillo, Rodriguez, and Ray (2014) reported that father connectedness was important in adolescents' suicidal ideation where low connectedness with father tend to induce suicidal thoughts among adolescents. Adolescents who have father who are supportive and give them approval for what they want to do in life are less likely to feel depressed and will not think of ending their life. 
Other than paternal approval, self-efficacy is another important factor in reducing the risk of developing suicidal ideation. Kobayashi, Fujita, Kaneko, and Motohashi (2015) who conducted a longitudinal study found that the participants developed suicidal ideation due to their lower level of self-efficacy. They concluded that individual with high self-efficacy was two times less likely to have suicide thoughts. This is because adolescents with high selfefficacy believe in themselves. They believe that they are able to overcome obstacles, cope with stressors and complete tasks that were given to them.

This study has identified two important factors namely self-efficacy and paternal approval as these factors significantly correlated with adolescents' suicidal ideation. Both of these variables is supported by the Social Cognitive Theory (Bandura, 1986) where both social and personal factor are important in reducing suicidal thoughts among adolescents. Adolescents who gain approval and supports from their father have higher self-efficacy where they believe in their capabilities. Thus, they are less likely to have suicidal thoughts as they believe they are able to overcome problems in life.

To conclude, gaining high approval from fathers helps in increasing adolescents' level of self-efficacy which in turn reduces their risk of having suicidal ideation. Parental approval may be a good fit in the Malaysian cultural context which emphasized child conformity, and subsequently strengthened the positive benefits of adolescents' belief in problem-solving and overcoming life challenges. Therefore, intervention dealing with adolescents' suicidal ideation should include parenting education for parents especially for fathers and also enhancing adolescents' self-efficacy.

Recommendation from this study for future research is to conduct longitudinal study in order to investigate the causes of suicidal ideation. Paternal approval and self-efficacy maybe the cause for the increase of suicidal adolescents in Asian countries, especially in Malaysia.

\section{References}

Bandura, A. (1977). Self-efficacy: Toward a unifying theory of behavioral change. Psychological Review, 84(2), 191-215.

Bandura, A. (1986). Social foundations of thought and action: A social cognitive theory. Englewood Cliffs, NJ: Prentice- Hall, Inc.

Buhrmester, D., \& Furman, W. (2009). The Network of Relationships Inventory: Relationship Qualities Version. Unpublished measure, University of Texas at Dallas.

Feng, J., Li, S., \& Chen, H. (2015). Impacts of stress, self-efficacy, and optimism on suicide ideation among rehabilitation patients with acute pesticide poisoning. PLoS ONE, 10(2), 1-11.

Gill, S. K., \& Gopal, N. D. (2010). Understanding Indian religious practice in Malaysia. Journal of Social Science, 25(1-3), 135-146.

Ibrahim, N., Amit, N., Che Din, N., \& Ong, H. C. (2017). Gender differences and psychological factors associated with suicidal ideation among youth in Malaysia. Psychology Research and Behavior Management, 28(10), 129-135. 
Kobayashi, Y., Fujita, K., Kaneko, Y., \& Motohashi, Y. (2015). Self-Efficacy as a suicidal ideation predictor: A population cohort study in rural Japan. Open Journal of Preventive Medicine, 5, 61-71.

Luca, S. M. D., Wyman, P., \& Warren, K. (2012). Latina adolescent suicidal ideations and attempts: Associations with connectedness to parents, peers and teachers. Suicide and Life-Threatening Behavior, 42(6), 672-683.

McNamara, P. M. (2012). Adolescent suicide in Australia: Rates, risk and resilience. Clinical Child Psychology and Psychiatry, 18(3), 351-369.

Nock, M. K., Borges, G., Bromet, E. J., Cha, C. B., Kessler, R. C., \& Lee, S. (2008). Suicide and suicidal behavior. Epidemiol Rev, 30(1), 133-154.

Osman, A., Gutierrez, P. M., Kopper, B. A., Barrios, F. X., \& Chiros, C. E. (1998). The Positive and Negative Suicide Ideation inventory: Development and validation. Psychological Reports, 82, 783-793.

Palkovitz, R., Trask, B. S., \& Adamsons, K. (2014). Essential differences in the meaning and processes of mothering and fathering: Family systems, feminist and qualitative perspectives. Journal of Family Theory \& Review, 6(4), 406-420. doi:10.1111/jftr.12048

Piña-Watson, B., Castillo, L. G., Rodriguez, K. M., \& Ray, S. (2014). Familial factors related to suicidal ideation of Latina adolescents in the United States. Archives of Suicide Research, $18(2), 213-220$.

Roslan, M. B. (2016). Suicide cases in Malaysia rather shocking. IIUM Today. Retrieved from http://news.iium.edu.my/2016/11/24/suicide-cases-in-malaysia-rather-shocking/

Schwarzer, R., \& Jerusalem, M. (1995). Generalized self-efficacy scale. In J. Weinman, S. Wright, \& M. Johntston, Measures in health psychology: A user's portfolio. Causal and control beliefs (pp. 35-37). Windsor, England: Nfer-Nelson.

Sipalan, J. (2012, June 5). Suicide rate on the rise in Malaysia. TheStar Online. Retrieved from http://www.thestar.com.my/News/Nation/2012/06/05/Suici de-rate-on-the-rise-inMalaysia.aspx/

Taliaferro, L. A., \& Muehlenkamp, J. J. (2014). Risk and protective factors that distinguish adolescents who attempt suicide from those who only consider suicide in the past year. Suicide and Life-Threatening Behavior, 44(1), 6-22.

Wan, G. W. Y., \& Leung, P. W. L. (2010). Factors accounting for youth suicide attempt in Hong Kong: A model building. Journal of Adolescence, 33, 575-582.

World Health Organization. (2018). Suicide data. Retrieved from http://www.who.int/mental_health/prevention/suicide/suicideprevent/en/

You, Z., Chen, M., Yang, S., Zhou, Z., \& Qin, P. (2014). Childhood adversity, recent life stressors and suicidal behavior in Chinese college students. PlosOne, 9(3), 1-7. 